\title{
Handling and Manipulation of Microcomponents: Work-Cell Design and Preliminary Experiments
}

\author{
Serena Ruggeri ${ }^{1}$, Gianmauro Fontana ${ }^{2}$, Claudia Pagano ${ }^{2}$, \\ Irene Fassi ${ }^{2}$, and Giovanni Legnani ${ }^{1}$ \\ ${ }^{1}$ Department of Mechanical and Industrial Engineering, University of Brescia, Brescia, Italy \\ \{serena.ruggeri, giovanni. legnani\}@ing.unibs.it \\ ${ }^{2}$ Institute of Industrial Technologies and Automation, CNR, Milan, Italy \\ \{gianmauro.fontana, claudia.pagano, irene.fassi\}@itia.cnr.it
}

\begin{abstract}
The paper introduces an experimental setup for the automatic manipulation of microcomponents, based on a 4 dof robot with Shoenflies motion and a two-camera vision system. The general architecture of the workcell is presented. The work-cell functionality was tested via repeatability experiments using a set of vacuum grippers. Due to their intrinsic simplicity, vacuum grippers are very cheap and appear a promising solution for micromanipulation. An innovative nozzle for a vacuum gripper was designed, fabricated and tested, comparing its performance with traditional needles. The design was conceived to reduce the frequency of occlusions of the gripper and handle a wide range of particles. The performed tests evaluate the success and precision of the part release. Indeed, this is a crucial aspect of micromanipulation because microparts tend to stick to the gripper preventing the successful performance of manipulation tasks. The results confirm that adhesive effects prevent the release of components. For this reason different strategies were adopted in order to improve the efficiency in the release of stuck components. This solution increases the percentage of release and, setting appropriately the intensity of the pressure, it does not affect negatively the accuracy nor the repeatability of the positioning.
\end{abstract}

Keywords: Micro-handling, Micro-Robotics.

\section{Introduction}

During the past decades several microproducts have been fabricated for a great variety of applications in the traditional fields and in more innovative areas. The majority of microsystems are currently fabricated by semiconductor-based manufacturing techniques, taking advantage of the consolidated experience gained from the fabrication of integrated circuits. More recently, a variety of emergent products (e.g. micro-resonators, radio frequency devices, drug delivery systems, chemical and biochemical sensors) requires components made of non semiconductor material in order to increase the performance and functionalities of the 
whole system. These components have to be made of metal, plastic and ceramic, which need appropriate fabrication processes and have to be assembled with each other or with semiconductor structures.

These hybrid three dimensional microproducts have still great difficulty in penetrating the market, mainly due to the limits of the fabrication processes that require manipulation and final assembly of microcomponents. However, new market perspectives can be reached automating the assembly phase. The main challenge is due to the new physical scenario when dealing with millimetric and sub-millimetric parts. Indeed, due to the high surface to volume ratio of microcomponents, superficial forces are predominant and the manipulation of microparts significantly differs from that of macroscopic ones. For this reason, the downscaling of standard assembling procedures at the microlevel is infeasible or inefficient and many operations have tobe done manually [1].

Many issues can affect one or more of the three main manipulation phases (grasp, handling, release) and have to be taken into account during the design of the microgripping systems. Due to the charging effects, the parts move into their energetically most favourable configuration, leading in an uncontrolled grasping or release. Moreover, due to the adhesion forces, objects stick to the gripper preventing the release that is not anymore facilitated by the gravitational force due to the reduced weight.

In the literature, several solutions to these manipulation problems can be found, including hybrid-type grippers where two principles are integrated in order to reduce the adhesive force during the release [2], grippers based on physical principles peculiar of the microscale that control and exploit the adhesive forces [3][4], and handling systems without physical contacts between the gripper and the component, in order to avoid stiction [5]. Among the strategies proposed for the micromanipulation there are: phase transition [6], magnetic [5], van der Waals [7], electrostatic [3][8], adhesive and capillary interactions [4][9]-[12] suction [13], and laser [14]. Each of these solutions has its own advantages and drawbacks, mainly concerning the cost, accuracy, repeatability, compliancy, versatility and complexity.

One of the strategies downsized from the macroworld is the use of the force generated by the pressure difference between the gripper and the atmosphere. Indeed, vacuum grippers are very common in the assembly of fragile macrocomponents and can be easily miniaturised. They can be very cheap as consist mainly of a micropipette connected to a vacuum pump. However, both the gripper and the part should have smooth surfaces to prevent air leakage. Moreover, like all the contact grippers at the microscale, the adhesion forces significantly affect the release so that the precise positioning is difficult to achieve. This is the reason why some expedients for the release of microcomponents have been proposed. For instance, a short pressure pulse can be applied to assist the release of the microcomponent, even if it can affect the accuracy of the positioning [13]. The adhesion due to the electrostatic force can be reduced coating the glass pipette for the suction with a conductive layer connected to the ground [15].

In this paper, an experimental setup devoted to the automatic manipulation of microcomponents is introduced and preliminary experiments on the grasping and 
releasing of parts are described and discussed. The performance of two standard vacuum microgrippers (commercially available needles for dispensing) with respect to an innovative multi-lumen nozzle (Fig. 1a) is critically analysed. The limitations of these grippers, mainly in the releasing phase, are highlighted and some solutions proposed.

\section{Experimental Setup}

A suitable experimental setup (Fig. 1) able to move the parts and measure their position in the working area was designed.

The work-cell is equipped with a Mitsubishi Electric RP-1AH robot (1). It presents a 5-joint closed link structure and 4 degrees of freedom with Schoenflies motion: 2 revolute joints for the positioning in the $x-y$ working area, a third revolute joint for rotation and a prismatic joint for the $\mathrm{z}$ vertical end-effector motion. The operating limits are $150 \times 105 \mathrm{~mm}^{2}$ with a vertical stroke of $30 \mathrm{~mm}$. The repeatability is $\pm 5 \mu \mathrm{m}$ in the $\mathrm{x}-\mathrm{y}$ plane, $\pm 10 \mu \mathrm{m}$ for the vertical motion and $\pm 0.02^{\circ}$ for the end-effector rotation.

A smart and standard mechanical interface (2) was realized in order to facilitate the tool change. It was directly connected to the bottom part of the hollow screw constituting the third and fourth axis of the robot.

The vacuum generation system is a critical part of the setup, mainly during the releasing phase. Two vacuum generation systems were tested. The former consisted of an air compressor, a FRL (Filter Regulator Lubricator) group, a normally closed solenoid valve mounted on the top of the robot arm and a piINLINE® Micro Ti vacuum ejector installed directly on the hose near the suction point. The latter used the same air compressor and FRL group, but was equipped with a piCOMPACT10 vacuum ejector (3). This ejector integrates a vacuum sensor and two normally closed solenoid valves, one for the supply and one for the release. This latter and more complex generation system was chosen to assist the release with a positive pressure. By modifying the throttling, the entity of the blow in the release phase can be set and optimized for the specific component and application.

The measurements of the position of the parts in the focal plane were performed using a suitable vision system, consisting of a camera (4) with field of view (FoV) $16.3 \times 13.5 \mathrm{~mm}^{2}$ and spatial resolution $6.6 \mu \mathrm{m}$ and a second camera (5) with FoV $25 \times 18.9 \mathrm{~mm}^{2}$ and spatial resolution $18.4 \mu \mathrm{m}$. The parts lied on a transparent glass substrate (6) so that the camera, fixed on a rigid structure below the robot working area, detects from the bottom their position and orientation. An opportune lighting system is essential for the detection, robust recognition and reliable measurement, thus a diffuse illumination of the scene was adopted, making the disturbance of the environment light negligible. In order to obtain better images, the end-effector was also equipped with a contrast panel (7) on the top of the gripping tool (8).

Concerning the gripping tools, the nozzle (Fig. 1a) has a central circular hole (radius $=55.4 \mu \mathrm{m}$ ) and an external circle (radius $=140.1 \mu \mathrm{m}$ ), circumscribing the internal hole and the other four holes. This geometry was conceived with the purpose of manipulating a wider range of components, avoiding the need of changing the tool for 
the manipulation of components different in size or geometry. The nozzle should be able to perform like a needle with dimension equal to its internal radius and a needle with dimension equal to its external one at the same time. Keeping that in mind, the performance of the nozzle was compared with the performance of two needles with internal diameters of 100 and $260 \mu \mathrm{m}$ (Fig. 1b).

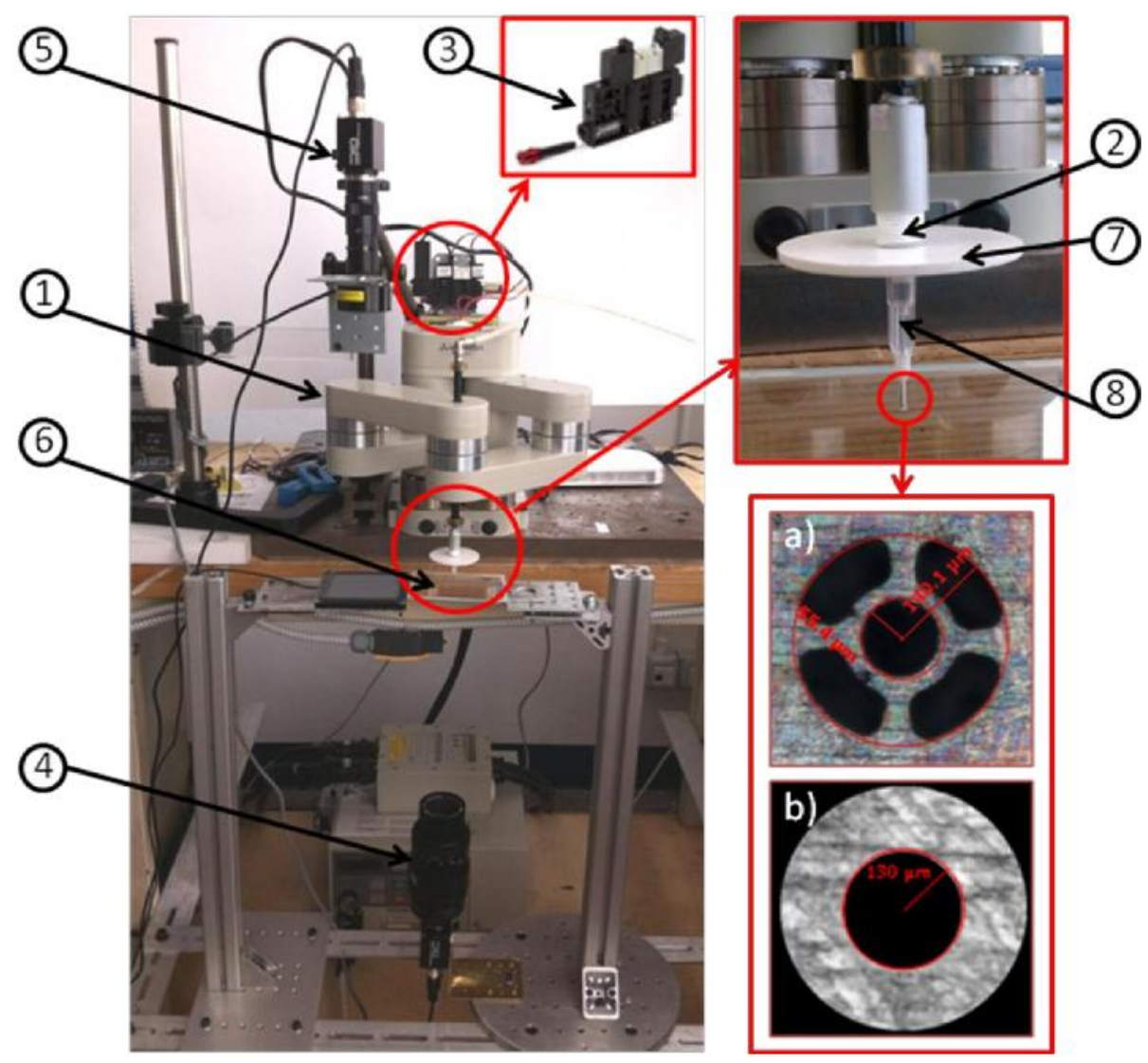

Fig. 1. Experimental setup

The camera and the robot were calibrated in order to allow the automatic part detection for the grasp and after the release and for the measure of the part position and orientation. A non-conventional calibration process was conceived and implemented in order to simultaneously calibrate the camera and georeference the camera with respect to the robot, without using external tools. This procedure was based on the use of a virtual grid with the same characteristics of the standard calibration pattern, created by moving a sphere gripped by the robot end-effector in the field of view of the camera. A similar procedure was presented in [16]. Thus, a suitable vision algorithm was developed in LabVIEW ${ }^{\circledR}$ for a complete calibration of the manipulation cell, compensating for perspective, distortion and spatial referencing 
errors. Moreover, a set of vision functions and image processing algorithms were implemented, including a robust pattern matching algorithm for the detection of the parts in the field of view and the elaboration of the displacement measurements.

\section{Experimental Procedures}

Repetitive tests of the grasping and releasing of microcomponents were carried out with the setup previously described. The initial tests showed that the results depended on the boundary conditions, for example the complexity of the trajectory of the robot during the handling of the components. Indeed, the release of the object was achieved more easily when the gripper performed a simple up and down movement, than if the pick and place motion includes a horizontal displacement, as if the gripper motion favoured the adhesion object-gripper. The tests were performed using a pick and place motion. For these preliminary experiments, the performance of each gripper was assessed using spheres with different diameters: $0.8 \mathrm{~mm}$ and $1.2 \mathrm{~mm}$ and for each sphere, a total of 30 grasp-motion-release cycles were performed. Moreover, three strategies for the release were adopted

a) the vacuum pump was switched off;

b) the robot moved brusquely $2 \mathrm{~mm}$ upwards;

c) the controller activated the release valve applying a positive pressure.

The feeding pressure was 2 bar, the vacuum flow $0.04 \mathrm{Nl} / \mathrm{s}$ and the execution time of the tests approximately 12 seconds. The environmental conditions were kept constant during all the tests: the temperature was $24{ }^{\circ} \mathrm{C}$ and the relative humidity $25 \%$. Given the size of the manipulated objects, a clean room environment was not necessary.

The releasing tests were performed approaching the sphere to the planar surface and releasing it from a distance of about $0.15 \mathrm{~mm}$. The precision (accuracy, repeatability) was estimated measuring the position of the sphere just before and after the release. To avoid undesired rolling of the sphere their shape and the horizontality of the plane were verified.

\section{$4 \quad$ Results and Discussion}

For each test cycle the accuracy (AC) and the repeatability (RP) were calculated. The accuracy AC is defined as the distance between the target position and the barycentre of the cloud of the actual achieved positions, while the repeatability RP is defined as the statistical positional deviation from the average, according to the standard ISO 9283. Although this standard is defined for the performance of manipulators, it was assumed significant also in this context.

The smallest needle (internal diameter $100 \mu \mathrm{m}$ ) was initially tested with both the spheres, but its dimension was so small that it grasped the component only if in contact with it. Since the contact could damage the component and the gripper, it was not considered for the present tests. A setup with an appropriate feedback should be developed in the future to allow a deeper investigation. In the following, the term 
needle will refer to the needle with internal diameter equal to $260 \mu \mathrm{m}$. In Table 1 the obtained results are shown. In the experiments exploiting the strategy $a$ described in section 3 the failure of the release was much more probable than the success. Despite of the low releasing percentage, for the sake of completeness, also the accuracy and repeatability of such tests are shown in the table.

Table 1. Accuracy, repeatability and release percentage of the two spheres by the two grippers

\begin{tabular}{|c|c|c|c|c|c|c|c|}
\hline \multirow{3}{*}{ Release } & \multirow{3}{*}{$\begin{array}{l}\text { Gripper } \\
\text { type }\end{array}$} & \multicolumn{6}{|c|}{ Sphere Dimension [mm] } \\
\hline & & \multicolumn{3}{|c|}{1.2} & \multicolumn{3}{|c|}{0.8} \\
\hline & & $\mathrm{AC}[\mathrm{mm}]$ & $\mathrm{RP}[\mathrm{mm}]$ & Release[\%] & $\mathrm{AC}[\mathrm{mm}]$ & $\mathrm{RP}[\mathrm{mm}]$ & Release[\%] \\
\hline \multirow{2}{*}{ 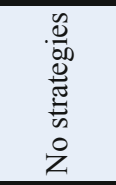 } & Needle & 0.283 & 0.913 & 13.33 & 0.106 & 0.438 & 16.67 \\
\hline & Nozzle & 0.181 & 0.380 & 16.67 & 0.382 & 1.524 & 16.67 \\
\hline \multirow{2}{*}{ 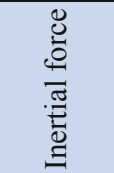 } & Needle & 0.175 & 5.094 & 96.67 & 0.775 & 4.992 & 43.33 \\
\hline & Nozzle & 0.402 & 2.791 & 40.00 & 0.383 & 3.053 & 70.00 \\
\hline \multirow{2}{*}{ 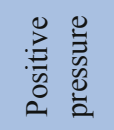 } & Needle & 0.026 & 0.263 & 90.00 & 0.027 & 0.199 & 100.00 \\
\hline & Nozzle & 0.030 & 0.330 & 96.67 & 0.098 & 0.589 & 56.67 \\
\hline
\end{tabular}

Regarding the comparison of the performance of the nozzle and the needle using the inertial force (par.3 strategy $b$ ) for the release, the results showed that the nozzle achieves lower percentage of release of the biggest sphere. This is probably due to the superficial force contribution of the area of the nozzle between the central hole and the external ones. The opposite is true for the smallest sphere; in this case the nozzle achieves a greater number of releases. This might be due to a smaller surface contact of the nozzle. Indeed, the surface contact between the needle and the sphere is always the same, regardless the dimension of the sphere. Instead, in the case of the nozzle, it is likely that the contact surface is smaller for the smallest sphere than for the biggest one, where all the external circle is probably in contact with the sphere. This could also be the reason why the nozzle showed a higher probability of releasing the smallest sphere compared to the biggest one.

Concerning the precision of the positioning, no great difference can be found for each of the two spheres, regardless the type of gripper. In the case of the needle the accuracy value is high, as well as the repeatability value. Therefore, even when the percentage of the release is very high the accuracy of the positioning is unacceptable for any precise application.

A more positive scenario appears with the nozzle, even if the results are still too inadequate for a microprecision manipulation. It is likely that the procedure can be 
improved in order to reduce the energy of the sphere after the release. This could reduce the displacement of the sphere after the detachment from the gripper. Nevertheless, all in all, as expected and suggested by the literature, this release strategy seems to be not suitable for precise manipulation.

In order to achieve better results the strategy $c$ was tested. It consists in assisting the release applying a small positive pressure. This strategy increases the percentage of release and, surprisingly it does not affect negatively the accuracy and repeatability of the positioning. However, the parameters that control the blow had to be optimized for each case; indeed the success of the experiments seemed to be significantly influenced by the chosen parameters, including the entity of the depression for the grasp and of the positive pressure for the release. A threshold of values, which allows the achievement of a high percentage of release and a great accuracy of the positioning was in some cases not found, mainly because of the rough regulation of the vacuum pump. In these cases, the experiments were done, trying to improve the precision rather than the success of the release. This strategy was preferred considering the future implementation of a control system able to indicate whether the release occurred or not. In any application such a system should be easily implementable, and preferable than dealing with a warranted but not precise release.

This issue occurred in the test of the nozzle and the small sphere, for which the achieved release percentage was lower than $60 \%$. In fact the release achieved with the nozzle seemed to be more unpredictable than the release using the needle. This might be due to the complex shape of the nozzle, so that uncontrolled air flows through its five holes could occur.

\section{Conclusions}

An experimental setup based on a robot, a vacuum generator system and a vision system was designed and developed. It allowed the automatic manipulation of microcomponents with a variety of vacuum grippers. The experiments were carried out comparing the performance of a standard vacuum gripper (a standard dispending needle) and a multi-lumen nozzle in grasping and releasing microspheres.

Two strategies were tested in order to achieve a correct and precise release, one based on the inertial force of the sphere and the other on the positive pressure. The percentage of release is significantly improved using the second strategy, even though the procedure for the optimization of the parameters is much longer.

In the case of the needle also the accuracy of the release was improved using the positive pressure release strategy, whereas the behaviour of the nozzle was unvaried, probably due to uncontrolled air flows through its five holes. The experiments showed that the performance of a traditional needle was higher than that of the nozzle. However, the nozzle seemed to be more flexible in handling components of various dimensions, whilst the needle size has to be chosen depending on the component dimensions.

Deeper research will be carried out on the nozzle, in order to understand the behaviour showed during the release with positive pressure, developing also some 
theoretical and numerical models. This will also help to identify if, for suitable dimensions of the spheres, the external holes can work as a mean of auto-centring of the sphere after the release.

\section{References}

1. Van Brussel, H., et al.: Assembly of Microsystems. Annals of the CIRP 49(2), 451-472 (2000)

2. Chen, T., et al.: A hybrid-type electrostatically driven microgripper with an integrated vacuum tool. Sensors and Actuators A: Physical 158(2), 320-327 (2010)

3. Hesselbach, J., et al.: Centering electrostatic microgripper and magazines for microassembly tasks. In: Proc. of SPIE, Microrobotics and Microassembly III, Newton, USA, October 29-30, vol. 4568, pp. 270-277 (1999)

4. Biganzoli, F., et al.: Development of a gripping system based on capillary force. In: Proc. of 6th IEEE ISATP, Montreal, Canada, July 19-21 (2005)

5. Gosse, C., Croquette, V.: Magnetic tweezers: Micromanipulation and force measurement at the molecular level. Biophys. J. 82(6), 3314-3329 (2002)

6. Kochan, A.: European project develops ice gripper for micro-sized components. Assembly Automation 17(2), 114-115 (1997)

7. Feddema, J.T., et al.: Micro-assembly planning with van der Waals force. In: Proc. of the IEEE International Symposium on Assembly and Task Planning, July 21-24, pp. 32-38 (1999)

8. Enikov, E.T., Lazarov, K.V.: Optically transparent gripper for microassembly. In: SPIE, vol. 4568, pp. 40-49 (2001)

9. Bark, C., Binneboese, T.: Gripping with low viscosity fluid. In: Proc. of IEEE Int. Workshop on MEMS, pp. 301-305 (1998)

10. Grutzeck, H., et al.: Downscaling of grippers for micro assembly. Microsystem Technologies 8, 27-31 (2002)

11. Obata, K.J., et al.: A scheme for micro-manipulation based on capillary force. J. Fluid Mech. 498, 113-121 (2004)

12. Saito, S., et al.: Capillary force with a concave probe-tip for micromanipulation. Applied Physics Letters 87(23), 234103-234103-3 (2005)

13. Zesch, W., et al.: Vacuum tool for handling microobjects with a NanoRobot. In: Proc. of ICRA, pp. 1761-1776 (1997)

14. Rambin, C.L., Warrington, R.O.: Micro-assembly with a focused laser beam. In: Proc. of IEEE MEMS, pp. 285-290 (1994)

15. Petrovic, D., et al.: Gripping tools for handling and assembly of microcomponents. In: Proc. of 23rd Int. Conf. on Microelectronics, vol. 1, pp. 247-250 (2002)

16. Tamadazte, B., et al.: Multiscale Calibration of a Photon Videomicroscope for Visual Servo Control: Application to MEMS Micromanipulation and Microassembly Sensors \& Transducers Journal 5(special issue), 37-52 (2009) 